\title{
Using Capillary Electrophoresis to Determine Acrylamide in Bakery Products
}

\author{
Kruchina-Bogdanov I.V. \\ AMT, Ltd \\ Saint-Petersburg, Russia \\ igogo011@gmail.com
}

\author{
Nilova L.P. \\ Peter the Great St. Petersburg Polytechnic University \\ Institute of Industrial Management, Economics and Trade \\ Saint-Petersburg, Russia \\ nilova1pp@mail.ru
}

Malyutenkova S.M.

Peter the Great St. Petersburg Polytechnic University Institute of Industrial Management, Economics and Trade Saint-Petersburg, Russia malutesha66@mail.ru

\author{
Naumenko N.V. \\ South Ural State University \\ School of Medicine and Biology \\ Chelyabinsk, Russia \\ naumenko_natalya@mail.ru
}

\begin{abstract}
The work objective is to identify the content of acrylamide in bakery products through capillary electrophoresis. For identification, we used the "Kapel 105-M" capillary electrophoresis system with spectrophotometric detection. The study was targeted at bakery products made of top-grade wheat flour by traditional formulations (without additives) with seabuckthorn powder added in the quantities of 5.0 and $10.0 \%$. Acrylamide was identified in the crust, in the layer under the crust and in the crumb of the products immediately after baking and after 24 hours of storage. Adding the sea-buckthorn powder in the amount of $5 \%$ into the bakery formulation decreases the acrylamide content in the crust and in the layer under the crust, and increasing its amount up to $10 \%$ causes formation of acrylamide with the maximum amount in the crust. In the course of storage of bakery products during 24 hours, the amount of acrylamide in bakery of traditional formulation with seabuckthorn powder additives decreases, especially in the crust and crumb.
\end{abstract}

Keywords—bakery products, acrylamide, capillary electrophoresis, sea-buckthorn powder

\section{INTRODUCTION}

Bread and bakery products pertain to food products in which dangerous substance - acrylamide with carcinogenic and toxic effects is formed at the final production stage baking, simultaneously with forming the sensory properties. Some monosaccharides and asparagine amino acid contained in fermented dough contribute to its formation; the temperature impact is higher than $200^{\circ} \mathrm{C}$ when baked; the water content decreases at the final baking stage $[1,2]$. The acrylamide content in bread and bakery products can vary within a large range - 22-3,436 $\mathrm{mg} / \mathrm{kg}$, depending on the formulation, being prevalent in grain and rye bread and bakery products with a high content of fats [3-5]. It is possible to reduce its formation by regulating the technological process (fermentation duration, baking temperature, amount of steam when baked, type of ovens), by selecting formulations (flour type and grade, fats, salt), and by using asparagine predecessors - the glycine and cysteine aminoacids [6-8].

The issue on the influence of plant components on acrylamide formation in bakery products remains unsolved. Plant components containing a complex of biologically active substances can both suppress and increase acrylamyde formation in bakery products, which refers to their quantitative and qualitative composition [9, 10]. Thus, the minimum acrylamide quantity in fried breadsticks is formed when 1 or $0.1 \%$ of bamboo leaf powder or green tea extract is added, respectively. What is more, bamboo leaf powder in the amount of 0.002 to $1 \%$ made a reducing effect, and the effect of 1 to $4.9 \%$ was augmenting. Such disposition was also admitted when green tea extract was used, but the acrylamide content started increasing when the extract was added in the amount exceeding $0.1 \%$ [11]. The main reason for this phenomenon is considered to be a very complex content of polyphenols contained in additives produced from vegetable raw materials. Polyphenols have different structures and functional groups which can react with acrylamide predecessors, with intermediate reaction links or with acrylamide itself, either by reducing its content or by contributing to its formation. Some of them can link carbonyl groups thus preventing acrylamide formation, while others will impede the acrylamide polymerization reaction in the baking process $[10,12,13]$. The acrylamide content as a final result of both positive and negative reactions depends on the prevalent reaction involving antioxidants, which can change with changes in the antioxidant concentration. The influence of the antioxidative activity (AOA) of the plant components on the decrease in acrylamide formation in bakery products remains controversial. For example, $2 \%$ cinnamon with AOA of $971.94 \mu \mathrm{mol}$ Trolox/g added into gibbery resulted in an increase in acrylamide formation by $29 \%$, and cloves with a 
lower AOA of $47.44 \mu \mathrm{mol}$ Trolox/g decreased it by $17.3 \%$. At the same time, coriander with AOA of $15.8144 \mu \mathrm{mol}$ Trolox/ $\mathrm{g}$ increased the acrylamide content in gibbery by $18.5 \%$ [14]. A certain role can be played by melanoidins also manifesting antioxidant properties, which can react at various stages of Maillard reaction [15].

The most widespread methods for identifying acrylamide in food products, including bread and bakery products, are the chromatographic methods $[5,6,14,16]$. Many authors consider a weak retention and deformation of the acrylamide peak in food products $[17,18]$ to be deficiencies of the chromatographic division. To increase the identification efficiency, the detection of bromo-derivatives of acrylamide on the electron capture detector may be used, which complicates such identification [16]. Using gas-liquid chromatography with mass-selective detection is more effective. But for efficient division, preliminary preparation of non-polar acrylamide derivatives, derivatization is required due to low concentrations in food products. Derivatization is time-consuming with a risk of analyte loss because of nonstability of the derivatives or due to interferences from the derivatization reagent $[19,20]$. A more simple and effective method for acrylamide identification is considered to be capillary electrophoresis, which, when combined with ultrasound acrylamide extraction from the food matrix into a water solution, makes it an environmentally friendly method $[21,22]$.

The work objective is to identify the acrylamide content by using the capillary electrophoresis method in different parts of bakery products (crust, under crust, crumb) of traditional formulations and with sea-buckthorn powder additives.

\section{MATERIALS AND METHODS}

\section{A. Preparation of bakery products}

Bakery products were produced by using the straight dough procedure, the weight was $100 \mathrm{~g}$, by the formulation, $\%$ : top-grade wheat flour (ash content $0.55 \%$; gluten $28.9 \%$ ); - 100; granulated sugar - 14.5; refined deodorized sunflower oil - 14.5; common salt - 1.5\%. In bakery products with sea-buckthorn additives, 5 and $10 \%$ of wheat flour was substituted with sea-buckthorn powder [23].

The bakery was made at a temperature of $220^{\circ} \mathrm{C}$ during 20 minutes. After the bakery products were cooled down to the room temperature, identical samples underwent the specimen preparation procedure at once and after storage in polymer film during 24 hours. The crust, the layer under the crust with a thickness of $1 \mathrm{~cm}$ and the central crumb were separated from the bakery products. Such different parts of the bakery products were dried separately in a cupboard drier at a temperature of $50^{\circ} \mathrm{C}$ until the constant weight was reached [21].

B. Preparation of samples for the test

Acrylamide was extracted from the component parts of the bakery products: $50 \%$ (by volume) with ethanol $-5 \mathrm{ml}$ per $300 \mathrm{mg}$ of the grinded air-dried sample after homogenization - in the ultrasonic bath $(250 \mathrm{Wt}, 18 \mathrm{kHz})$ at $20^{\circ} \mathrm{C}$ during 30 min. The extract was decanted and clarified by centrifugation (10 min., $3000 \mathrm{~g}$ ).

C. Preparation of the instrument for operation

For acrylamide identification, the "Kapel 105 M" capillary electrophoresis system was used (NPF Lumex OJSC, Russia) with a quartz capillary having a diameter of $75 \mu \mathrm{m}$ and length of $50 / 60 \mathrm{~cm}$ (effective/total). The capillary was washed before the sample was injected: $0.5 \mathrm{M} \mathrm{HCl}-5 \mathrm{~min}$., water -5 min., $0.5 \mathrm{M} \mathrm{NaOH}-5 \mathrm{~min}$., water $-5 \mathrm{~min}$., supporting dectrolyte $-10 \mathrm{~min}$. $50 \mathrm{mM}$ of sodium tetraborate, $\mathrm{pH} \mathrm{9.2,}$ was used as supporting electrolyte.

The capillary was thermostated at a temperature of $20^{\circ} \mathrm{C}$ during 10 minutes. Detection was carried out at $202 \mathrm{~nm}$. The detection threshold (signal: noise $=3: 1$ ) was $90 \mu \mathrm{g} / \mathrm{kg}$. Hydrodynamic sample injection: $50 \mathrm{mbar} / 4 \mathrm{~s}$.

Electrophoresis was conducted under the voltage of $10 \mathrm{kV}$. E-O converter marker (electroosmotic flow) - benzyl alcohol (99.9\%), UAHIM, Russia. Acrylamide produced by Reanal, Budapest, Hungary, was used as reference substance.

D. Data processing

The mass concentration of the component in the sample under analysis $(\mathrm{X})$ was calculated by the formula:

$$
x=(k \cdot C) / m
$$

where $k$ is the sample dilution coefficient; $C$ is the acrylamide concentration determined by using the calibration graph, $\mu \mathrm{g} ; m$ - sample weight, $\mathrm{g}$.

The measurement result is represented in the following form: $\mathrm{X} \pm \Delta \mu \mathrm{g} / \mathrm{kg}$ ( $\mathrm{X}$ is the concentration of the component in the sample, $\mu \mathrm{g} / \mathrm{kg} ; \Delta$ - the absolute error range in the identification, $\mathrm{mg} / \mathrm{g}$, with the confidential probability of $\mathrm{P}=$ 0.90). The arithmetic mean of the results from three parallel identifications was taken as the final result of the test.

\section{RESULTS AND DISCUSSION}

A. Plotting the calibration graph

To plot the calibration graph, a series of tests was performed on water solutions with different acrylamide concentrations.

The acrylamide samples were prepared on the microbalance C-31 Microbalance /CAHN, USA; accuracy class 7 .

On electropherograms of acrylamide water solutions, irrespective of its concentration, the acrylamide peak was recorded on the 19th minute (Fig.1,2,3).

The relative standard deviation between the measurement series was - $10.2 \%$.

On the basis of the data obtained, a calibration curve was plotted (Fig.4), which had a linear dependence and complied with the equation: $\mathrm{y}=0.0015 \mathrm{X}, \mathrm{R}_{2}=0.9823$. Method linearity — up to $3,000 \mu \mathrm{g} / \mathrm{kg}$.

B. Acrylamide identification in bakery products

In bread and bakery products, acrylamide is formed uniformly due to different heating modes for dough intermediates and different water loss in crust and crumb formation during the baking process $[1,2]$. 


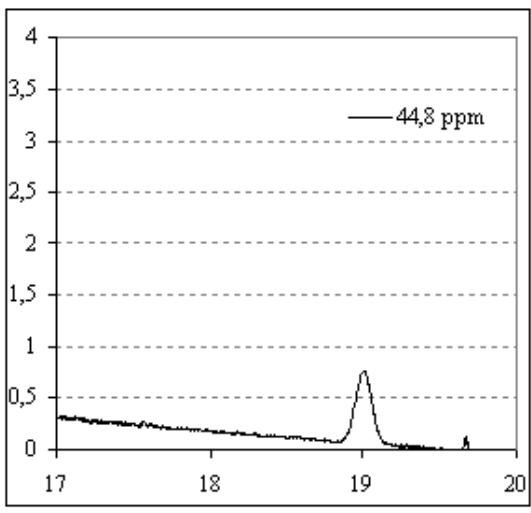

Fig.1. Electropherograms of acrylamide (AA) water solutions in concentrations $44,8 \mathrm{ppm}$.

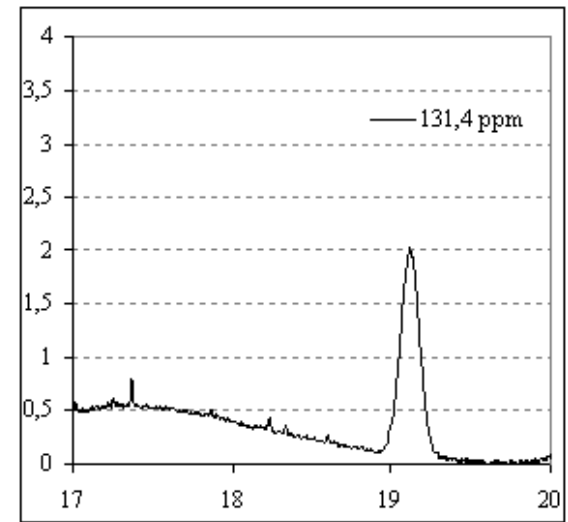

Fig.2. Electropherograms of acrylamide (AA) water solutions in concentrations $131,4 \mathrm{ppm}$.

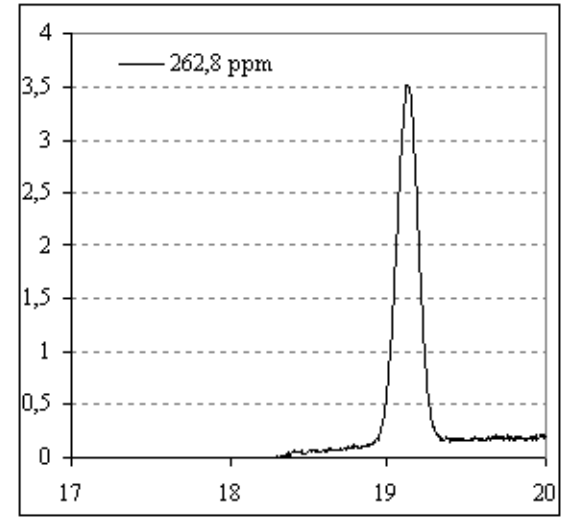

Fig.3. Electropherograms of acrylamide (AA) water solutions in concentrations of $262,8 \mathrm{ppm}$.

A small molecular mass of acrylamide with high solubility in water, combined with irregular distribution of water in bakery crumbs, with water migration during storage, enables us to suppose acrylamide migration. To confirm the supposed acrylamide migration in bakery products, the tests were carried out both in the crust, crumb and in the layer under the crust after cooling and after 24 hours of storage in package. The plant components (powders, extracts) added to bakery products, depending on their concentration, can either suppress or cause acrylamide formation in them $[10,11,13]$.
Thus, the acrylamide amount was identified in bakery products enriched with sea-buckthorn powder in different quantities -5 and $10 \%$.

The electropherograms of bakery samples made by traditional formulations with sea-buckthorn powder had approximately the same appearance (Fig. 5,6,7), both for the crust and crumb, and for the layer under the crumb, and the recorded peaks differed in their area. The results of the acrylamide content in the objects under analysis are presented in Table 1 .

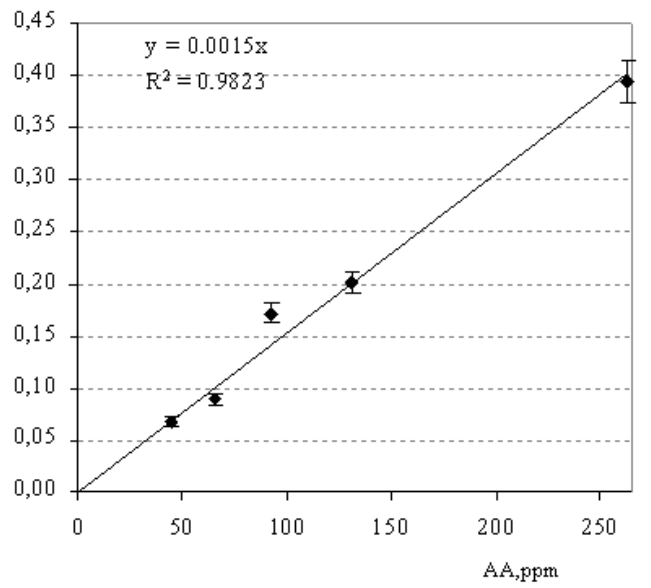

Fig.4. Calibration curve.

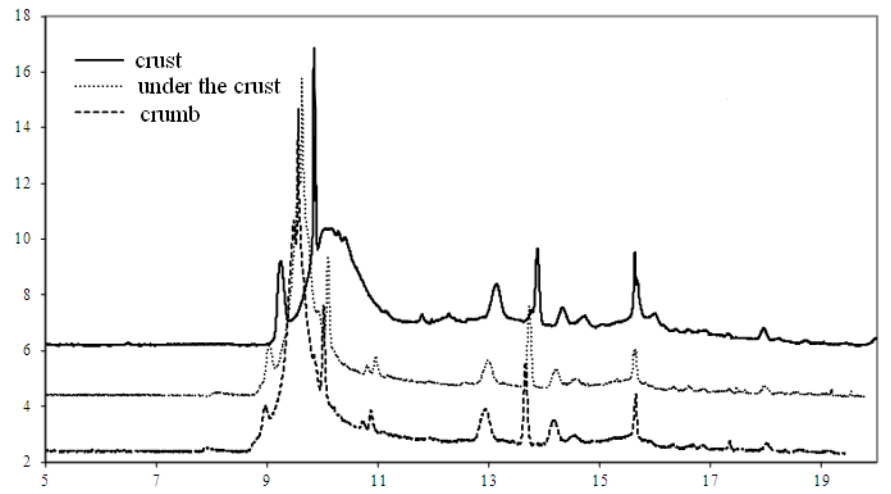

Fig.5. Electropherograms of component parts in bakery products of traditional formulations.

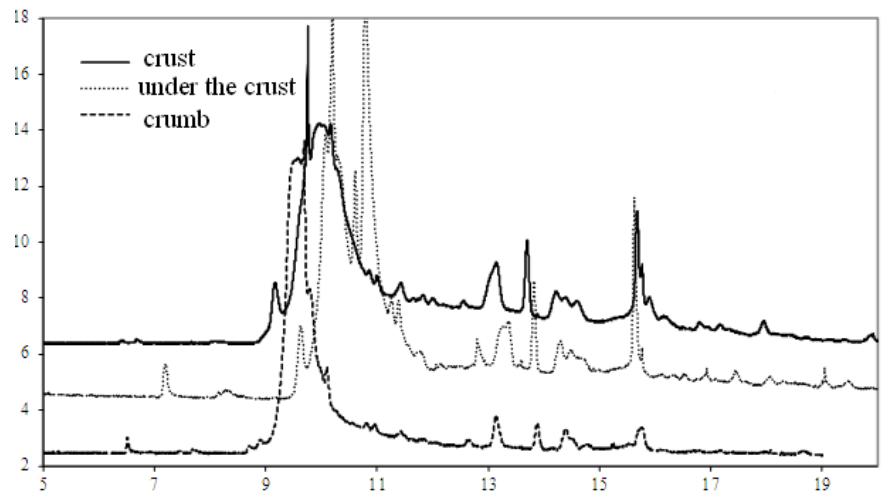

Fig.6. Electropherograms of component parts in bakery products with $5 \%$ of sea-buckthorn powder. 


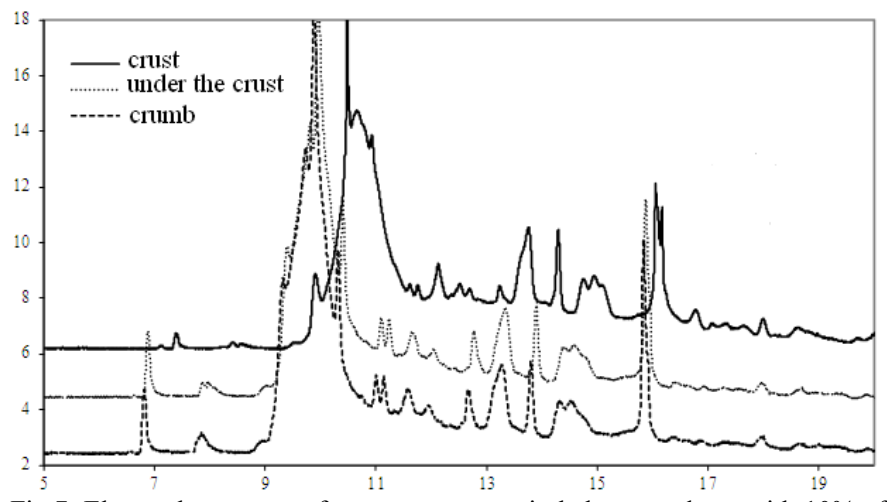

Fig.7. Electropherograms of component parts in bakery products with $10 \%$ of sea-buckthorn powder.

In all the samples under analysis, minor peaks of different intensities were recorded on the 19th minute (Fig.8). Their intensity varied depending on the formulation and component part of the bakery product. The results of the acrylamide content in the objects under analysis are presented in Table 1.

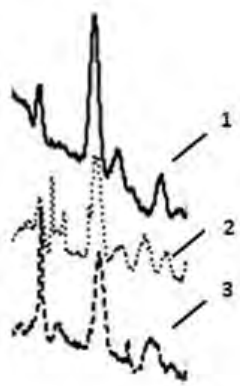

Control

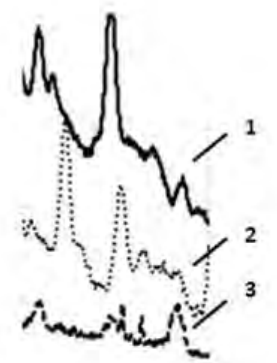
With sea-buckthorn powders
$\mathbf{5} \%$

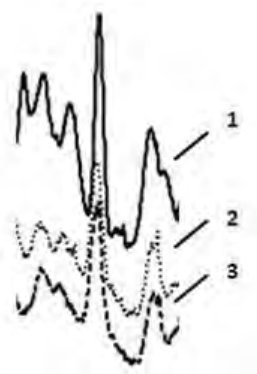

With sea-buckthorn powders $10 \%$
Fig.8. Enlarged fragments of the acrylamide peaks on electropherograms for the component parts of bakery products (components of bakery products: 1 crunch; 2 - under the crunch; 3 - crumb).

TABLE I. CONTENT OF ACRYLAMIDE (ACR) IN BAKERY PRODUCTS

\begin{tabular}{|c|c|c|c|}
\hline \multirow{2}{*}{ Bakery products } & \multicolumn{3}{|c|}{$\mathrm{ACR}, \mu \mathrm{g} / \mathrm{kg} \mathrm{DM}^{\mathrm{a}}$} \\
\hline & Crust & Under crust & Crumb \\
\hline control & $246.1 \pm 21.0$ & $213.6 \pm 20.8$ & $244.5 \pm 21.2$ \\
\hline $\begin{array}{l}\text { with } \\
\text { sea-buckthorn } \\
\text { powder } 5 \%\end{array}$ & $186.8 \pm 15.0$ & $91.4 \pm 8.8$ & $290.7 \pm 23.8$ \\
\hline $\begin{array}{l}\text { with } \\
\text { sea-buckthorn } \\
\text { powder } 10 \%\end{array}$ & $1,153.4 \pm 29.0$ & $517.5 \pm 28.0$ & $503.9 \pm 27.6$ \\
\hline
\end{tabular}

a. Data are expressed as mean \pm standard deviation $(n=3)$ on a dry matter basis.

The acrylamide content in different component parts of the bakery products cooked by traditional formulations was almost the same immediately after baking, especially between the crust and the crumb. A smaller amount of acrylamide is fixed under the crust layer on average by $13 \%$ compared to bark or crumb. The variations stayed within the experiment error range. Higher acrylamide content was recorded in comparison with wheat bread, as shown in the research works of other authors $[5,6]$. The obtained acrylamide values stayed within the same limits as for breadsticks made of wheat flour for cooking which fats are used [11]. That confirms the influence of fats in the formulation and the weight of products on acrylamide formation.

More considerable changes in the component parts of bakery products were obtained after adding sea-buckthorn powder in different quantities. In bakery products with the addition of sea-buckthorn powder in amounts of $5.0 \%$ acrylamide prevailed in the crumb $-290.7 \mu \mathrm{g} / \mathrm{kg}$ and in the crust $-186.8 \mu \mathrm{g} / \mathrm{kg}$. The smallest number recorded in the subcortical layer is $91.4 \mu \mathrm{g} / \mathrm{kg}$. Differences in the amount of acrylamide between the parts ranged from 1.5 times between crust and crumb up to 3 times between crumb and subcortical layer in comparison with bakery products of the traditional formulation

Adding 5\% of sea-buckthorn powder in bakery products caused a decrease in acrylamide formation in the crust and under the crust 1.32 and 2.33 times, respectively. At the same time, its amount in the crumb was higher than in bakery products of traditional formulations - by $18.9 \%$.

An increase in the amount of sea-buckthorn powder up to $10 \%$ caused acrylamide increase in the bakery products, which raised its amount $4.9 ; 2.2 ; 2.6$ times, respectively, for the crust, the layer under the crust and the crumb as compared with the reference samples. It was found that the amount of acrylamide in the crust increased 6 times, in the subcortical layer -5.7 times, in the crumb - 1.7 times compared with bakery products with $5 \%$ of the sea-buckthorn powder. Most of all acrylamide was formed in a crust $-1,153.4 \mu \mathrm{g} / \mathrm{kg}$. But whereas the acrylamide formation was uniform in the reference, it almost twice prevailed in the crust in the bakery products with $10 \%$ sea-buckthorn powder, where it was uniform under the crust and in the crumb. That proves the data of the other authors $[10,11,25]$ that a high amount of polyphenols in plant components may cause an increase in acrylamide formation in bakery products.

After 24 hours of keeping the samples in polymer film, changes occurred in the acrylamide content (Table 2).

TABLE II. CONTENT OF ACRYLAMIDE (ACR) IN BAKERY PRODUCTS IN 24 HOURS AFTER BAKING

\begin{tabular}{|l|c|c|c|}
\hline \multirow{2}{*}{ Bakery products } & \multicolumn{3}{|c|}{ ACR, $\boldsymbol{\mu g} / \mathbf{~ k g ~ D M}^{\mathbf{b}}$} \\
\cline { 2 - 4 } & Crust & Under crust & Crumb \\
\hline control & $85.2 \pm 8.0$ & $121.7 \pm 10.8$ & $55.8 \pm 21.2$ \\
\hline $\begin{array}{l}\text { with sea-buckthorn } \\
\text { powder 5\% }\end{array}$ & $61.1 \pm 5.0$ & $84.2 \pm 8.0$ & $65.0 \pm 5.9$ \\
\hline $\begin{array}{l}\text { with sea-buckthorn } \\
\text { powder 10\% }\end{array}$ & $56.9 \pm 5.0$ & $251.5 \pm 10.0$ & $275.7 \pm 17.5$ \\
\hline \multicolumn{4}{|c}{ b. Data are expressed as mean \pm standard deviation $(n=3)$ on a dry matter basis. }
\end{tabular}

In bakery products of traditional formulation, the amount of acrylamide was $121.7 \mu \mathrm{g} / \mathrm{kg}$ under the crust and $55.8 \mu \mathrm{g} /$ $\mathrm{kg}$ in the crumb. After the storage of bakery products, the amount of acrylamide decreased 1.8 times times under and 4.4 times in the crumb compared to freshly baked bakery products. Before the storage of bakery products, acrylamide predominated in the crust and crumb, and after storage - under the crust. It may be associated with water migration from the internal crumb layers to the external ones during the bakery 
drying process, and with their subsequent volatilization from the surface.

The same tendency (reduction in the amount of acrylamide in bakery products after storage) was recorded for the bakery products with sea-buckthorn powder, with no dependence on its amount. Acrylamide was contained in both the crumb and under the crust in bakery products with sea buckthorn powder added in the quantities of $5.0 \%$ than in bakery products with sea buckthorn powder added in the quantities of $10 \%$. Acrylamide prevailed in the subcortical layer of bakery products with $5 \%$ of sea-buckthorn powder, and in the crust and crumb its amount was almost the same. Acrylamide prevailed in the crumb in bakery products with $10 \%$ of seabuckthorn powder, compared with the subcortical layer and crust. The difference in the amount of acrylamide in the crumb and subcortical layer was insignificant, the statistical significance was absent. This could be due to the prevalence of water bound by biopolymers of sea-buckthorn powder, which prevented the migration of acrylamide along with water [25]. At the same time, in the crust of bakery products with $10 \%$ of sea-buckthorn powder, the amount of acrylamide was minimal compared to all the samples studied and was only $56.9 \mu \mathrm{g} / \mathrm{kg}$.

After 24 hours of storage in the reference crumb sample and in the sample with 5\% sea-buckthorn powder, the decrease in the acrylamide amount was the same -4.4 times, and in the sample containing $10 \%$ sea-buckthorn powder, the decrease was only 1.8 times. In the crust - vice versa, the greatest decrease in acrylamide was determined in the bakery products containing $10 \%$ sea-buckthorn powder -20 times for 24 hours of storage.

\section{CONCLUSIONS}

The quantitative determination of acrylamide in bakery products can be carried out by capillary electrophoresis. The relative standard deviation between the series of measurements is $10.2 \%$. It was determined that acrylamide formation in bakery products depends on their formulations. Adding sea-buckthorn powder in the amount of 5\% into the bakery formulation reduces the acrylamide content in the crust and under the crust, and an increase in its quantity up to $10 \%$ contributes to acrylamide formation with its maximum amount in the crust. In the course of storage of bakery products during 24 hours, the acrylamide amount in bakery products of traditional formulations and in those with sea-buckthorn additives decreases, especially in the crust and crumb.

\section{References}

[1] J. Keramat, A. LeBail, C. Prost and M. Jafari, "Acrylamide in Baking Products: A Review Article,” J. Food Bioprocess Technol, 2011, vol. 4. pp. 530-543.

[2] L. Ahrné, C.G. Andersson, P. Floberg, J. Rosén and H. Lingnert, "Effect of crust temperature and water content on acrylamide formation during baking of white bread: Steam and falling temperature baking," J.LWT Food Science and Technology, 2007, vol. 40, №10, pp.1708-1715.

[3] O.V. Bagryantseva, G.N. Shatrov and S.A. Khotimchenko, "Acrylamide. Its synthesis in processed food and ways of problem solution,” J. Voprosy Pitaniia, 2010, vol.79, №1, pp. 4-12.
[4] U.S. FDA. Survey Data on Acrylamide in Food: Individual Food Products.URL:

http:/www.fda.gov/Food/FoodborneIllnessContaminants/Chemical Contaminants/ucm053549.htm\#u1004. (Date: 20.06.2018)

[5] A. Horszwald, F.J. Morales, M.D. Castillo and H. Zielinski, "Evaluation of antioxidant capacity and formation of processing contaminats during rye bread making," J. of Food and Nutrition Research, 2010, vol. 49 (3), pp. 149-159.

[6] M. Przygodzka, M.K. Piskula, K. Kukurov, Z. Ciesarov, A. Bednarikova and H. Zielinski "Factors influencing acrylamide formation in rye, wheat and spelt breads," J. of Cereal Science. 2015, vol. 65. pp. 96-102.

[7] A. Claus, M. Mongili, G. Weisz, A. Schieber and R. Carle, "Impact of formulation and technological factors on the acrylamide content of wheat bread and bread rolls,” J. of Cereal Science, 2008, vol.47, №3, pp.546-554.

[8] M.N.S. Kumar, C.A. Shimray, D. Indrani and H. K. Manonmani, "Reduction of Acrylamide Formation in Sweet Bread with LAsparaginase Treatment,” J. Food Bioprocess Technol, 2014, vol. 7 (3). pp.741-748.

[9] Yu.G., Bazarnova and O.B. Ivanchenko, "Investigation of the composition of biologically active substances in extracts of wild plants," J. Voprosy Pitaniia, 2016, vol.85, №5, pp.100-107.

[10] Y. Liu, P. Wang, F. Chen, Y. Yuan, Y. Zhu and H. Yan, "Role of plant polyphenols in acrylamide formation and elimination," J. Food Chemistry, 2015, vol.186, №1, pp.46-53.

[11] Y. Zhang and Y. Zhang, "Study on reduction of acrylamide in fried bread sticks by addition of antioxidant of bamboo leaves and extract of green tea," Asia Pac J Clin Nutr., 2007, vol.16. pp. 131-136.

[12] Yu.G. Bazarnova, "Study of content of some food supplements with antioxidant activity in wild herbs and berries,"J. Voprosy Pitaniia, 2007, vol.76, №1, pp. 22-26.

[13] M. Huang, Q. Wang. X. Chen, and Y. Zhang, "Unravelling effects of flavanols and their derivatives on acrylamide formation via support vector machine mode," J. Food Chemistry, 2017, vol. 221, №15. pp. 178-186.

[14] L. Marková, Z. Ciesarová, K. Kukurová, H. Zielinski, M. Przygodzka and A. Bednáriková, "Influence of various spices on acrylamide content in buckwheat ginger cakes,” J. Chemical Papers, 2012, vol. 66, pp.949954.

[15] L.P. Nilova and Pilipenko T.V. "Evaluation of antioxidant properties of enriched bakery products in experiment on laboratory animals," J. Voprosy Pitaniia, 2016, vol.85, №6, pp. 39-47.

[16] V.V. Bessonov, A.D. Malinkin, O.I. Perederyaev, M.N. Bogachuk, S.V. Volovich and Yu.V. Medvedev, Development of methods for determining acrylamide in food products by gas-liquid chromatography," J. Voprosy Pitaniia, 2011, vol. 80, №4, pp. 79-83.

[17] J. Oracz, E. Nebesny and D. Zyzelewicz, "New trends in quantification of acrylamide in food products," J.. Talanta, 2011, vol.86, pp.23-34.

[18] Y. Zhang, H. Fang, and Y. Zhang, "Study on formation of acrylamide inasparagine-sugar microwave heating systems using UPLC-MS/MS analytical method," J. Food Chemistry, 2008, vol.108, pp.542-550.

[19] Y. Zhang, Y. Ren, J. Jiao, D. Li and Y. Zhang, "Ultra high-performance liquid chromatography-andem mass spectrometry for the simultaneous analysis of asparagine, sugars, and acrylamide in Maillard reactions," J. Analytical Chemistry, 2011, vol. 83, pp. 3297-3304.

[20] N.G. Halford, N. Muttucumaru, S.J. Powers, P.N. Gillatt, L. Hartley, J.S. Elmore et al., "Concentrations of free amino acids and sugars in nine potato varieties: Effects of storage and relationship with acrylamide formation," J. of Agricultural and Food Chemistry, 2012, vol. 60, pp.12044-12055.

[21] D. El-Hady and H. M.Albishri, "Simultaneous determination of acrylamide, asparagine and glucose in food using short chain methyl imidazolium ionic liquid based ultrasonic assisted extraction coupled with analyte focusing by ionic liquid micelle collapse capillary electrophoresis," J. Food Chemistry, 2015, vol.188, pp.551-558.

[22] E. Bermudo, O. Núñez, L. Puignou and M.T. Galceran, "Analysis of acrylamide in food products by in-line preconcentration capillary zone electrophoresis," J. of Chromatography A, 2006, vol.1129, № 1, pp.129. 134. 
[23] L. Nilova and S. Malyutenkova, "The possibility of using powdered seabuckthorn in the development of bakery products with antioxidant properties,” J. Agronomy Research, 2017, vol.16, № SII, pp. 1444-1456.

[24] R.V. Hedegaard, K. Granby, H. Frandsen, J. Thygesen and L.H Skibsted, "Acrylamide in bread. Effect of prooxidants and antioxidants," J. Eur Food Res. Technol, 2008, vol. 227, pp. 519-525.
[25] S. Palyvoda, V. Urchak, T. Golikova and V. Fomenko, "Study of the mechanism of the retarding effect on bread staling by using natural gums,’J. Food and Environment Safety, 2013, vol.4, №12, pp.300-310. 